\title{
Fractional Fourier Transform of Boehmians
}

\author{
S.B. Gaikwad ${ }^{1}$ \\ P.G. Department of Mathematics, New Arts Comm. \& Sci. College Ahmednagar \\ Dist: Ahmednagar, 414001,(M.S) India.
}

\begin{abstract}
In this paper we introduce two spaces of Boehmians each of which contains the dual of a certain space of entire functions. Both these spaces of Boehmians are shown to be isomorphic to each other under the Fractional Fourier transform. We extend the theory of the Fractional Fourier transform on this new Boehmians space.

Mathematics Subject Classification: (2000) 44A15, 44A40, 46F12, 44-99.

Key Words and Phrases: Boehmians, Convolution, entire function, Fractional Fourier transform, Tempered distributions.
\end{abstract}

\section{Introduction}

The fractional Fourier transform (fractional FT) $\mathrm{R}^{\alpha}$ is an extension of the ordinary Fourier transform and depends on a parameter $\alpha$ that can be interpreted as a sort of rotation by an angle $\alpha$ in the position frequency plane Alieva \& Barbe [1]. Pathak

[21-22] gave the comprehensive account of the Fourier transform on various spaces of distributions including distribution of the compact support.

The theory of Boehmians was motivated by the concept of regular operator's [15]. The notion of convergence on the space of Boehmians and their properties are developed in [16]. Further, various types of Boehmians are introduced and investigated in $[7,8,9,10,11,12,13,14,18,19]$. Integral transforms for several of these Boehmians space are defined and their properties studied in $[8,9,10,13,14,18]$.

We wish to mention that in the literature so far developed spaces of complex valued functions of a real variable \& their duals are generalized to the spaces of Boehmians and the classical theory of Fractional transform was also extend. However, the images under the Fractional Fourier transform were invariably classical spaces of distributions only.

On the other hand, Howell [3-6] introduces "new" theory of Fourier analysis, this presenting an entirely a new approach. For this purpose, the space G, conceived by Howell [3] of rapidly decreasing test functions is considered. The testing function space $\mathrm{G}$ consisting of entire functions. He also develops the theory of FT on $\mathrm{G}$ and its dual $G^{\prime}{ }^{\prime}$ in a series papers [4-6]. Howell, even proves that $\mathrm{G}$ is dense in $\mathrm{S}$.

It is because of this that the theory of Fractional Fourier transform on $G^{\prime}$ and hence also on $B\left(G^{c}, \Delta\right)$ is more general than the theory of Fractional FT on classical tempered distributions.

In this paper we introduce two spaces of Boehmians each of which contains the dual space $G^{\prime}$ described above \& extend the theory of Fractional Fourier transform. Moreover, this extended Fractional Fourier transform can now be considered as a continuous linear isomorphic between these two spaces of Boehmians.

In section 2 we shall recall various spaces of analytic functions and their duals available in the literature. The definitions of Fractional FT, convolution of Fractional FT has been defined. We shall also refer to the literature for all the results we require as preliminaries.

In section 3 we shall recall the general construction of Boehmians is given in [16]

We also introduce two spaces of Boehmians \& investigate the convergence properties in these Boehmians. Further, we also demonstrate how the classical dual space can be viewed as a dense subspace of both these spaces of Boehmians.

In the section 4, we shall define the Fractional FT \& prove that the two spaces of Boehmians are in some sense isomorphic to each other under the Fractional FT.

\section{Preliminaries}

Throughout this paper, $\mathrm{n}$ will denote some arbitrary fixed positive integer and $\wedge$ will denote the Lebesgue measure on $\mathfrak{R}$. We use $\mathrm{N}, \mathrm{N}_{0}, \mathfrak{R}, \varnothing$ to denote the positive integers, non-negative integers, real numbers and the complex numbers respectively.

Two spaces of functions will be of special importance. They will be denoted by testing functions spaces $\mathrm{G} \& \boldsymbol{G}^{\boldsymbol{c}}$ Here we shall recall the definition of testing function spaces $\mathrm{G} \& G^{\prime}$ defined in [3-6]. 
Let $B_{v}=\{z \in \varnothing:|\operatorname{Imz}| \leq v\}$

DEFINITION 2.1: A function $\phi: \phi \rightarrow \phi$ is an element of $\mathrm{G}$ if and only if it satisfies the two conditions.

(i) $\phi$ is an analytic function of each complex variable

(ii) For every $\gamma>0$,

$$
\sup _{z \in B_{\gamma}}\left\{e^{\gamma|\operatorname{Re} z|}|\phi(z)|\right\}<\infty .
$$

DEFINITION 2.2: A function $\phi: \phi \rightarrow \phi$ is an element of $\boldsymbol{G}^{c}$ if and only if it satisfies the conditions, (i) $\mathrm{f}$ is an entire function.

(ii) There is a fixed $\gamma \geq 0$ such that for each $\delta>0,\|f\|_{v}=\sup _{z \in B_{v}}\left\{e^{-\gamma|\operatorname{Re} z|}|f(z)|\right\}$

From the above two definitions it is clear that $G \subset G^{c}$. The topology on $\mathrm{G}$ is defined by the set of seminorms

Where

DEFINITION 2.3: The Fractional Fourier Transform: The one dimensional Fractional FT with parameter

$$
\begin{gathered}
\left\{\|-\|_{\gamma} / \gamma>0\right\} \\
\|\phi\|_{\gamma}=\sup _{z \in B_{\gamma}}\left\{e^{\gamma|\operatorname{Re} z|}|\phi(z)|\right\} . \\
{\left[R^{\alpha} f(x)\right](\xi)=F_{\alpha}(\xi)=\int_{-\infty}^{\infty} K_{\alpha}(x, \xi) f(x) d x---(1)}
\end{gathered}
$$

$\alpha$ of $f(x)$ denoted by $R^{\alpha} f(x)$ [1], performs in linear operation, given by the integral transform

Where the kernel

$$
K_{\alpha}(x, \xi)=(2 \pi i \operatorname{Sin} \alpha)^{\frac{-1}{2}} \exp (i \alpha / 2) \exp \left(\frac{i}{2 \operatorname{Sin} \alpha}\left(\left(x^{2}+\xi^{2}\right) \operatorname{Cos} \alpha-2 x \xi\right)\right)
$$

This kernel can be written as

$$
\begin{aligned}
& K_{\alpha}(x, \xi)=C_{1 \alpha} \exp \left(i C_{2 \alpha}\left(\left(x^{2}+\xi^{2}\right) \operatorname{Cos} \alpha-2 x \xi\right)\right) \\
& =C_{1 \alpha} \exp \left(i C_{2 \alpha} x^{2} \operatorname{Cos} \alpha\right) \exp \left(i C_{2 \alpha} \xi^{2} \operatorname{Cos} \alpha\right) \exp \left(-2 i C_{2 \alpha} x \xi\right) \\
& \text { where } C_{1 \alpha}=(2 \pi i \operatorname{Sin} \alpha)^{-1 / 2} \exp (i \alpha / 2) \text { and } C_{2 \alpha}=\frac{1}{2 \operatorname{Sin} \alpha} . \\
& \qquad C_{1 \alpha}=(2 \pi i \sin \alpha)^{-1 / 2} \exp (i \alpha / 2) \text { and } \\
& \qquad C_{2 \alpha}=\frac{1}{2} \sin \alpha .
\end{aligned}
$$

$\mathrm{K}_{\alpha}(\mathrm{x}, \xi)$ is the propagator of the non-stationary Schrodinger equation for a harmonic oscillator, which is well know in quantum mechanics (where $\alpha=\omega t$ relates to time $t$ and classical frequency $\omega$, and $\xi$ is a position at the moment $\mathrm{t}$ ). Changing gradually the angle $\alpha$ the fractional FT permits to input function $\mathrm{f}(\mathrm{x})$ to its Fourier image $F_{/ 2}(\xi)$ for $\alpha=\pi / 2$, then to $F(-x)$ for $\alpha=\pi$ and to $F_{\pi / 2}(-\xi)$ for $\alpha=3 \pi / 2$. Remark [2]:

The fractional FT at angle equal to $2 \pi \mathrm{n}$ ( $\mathrm{n}$ is an integer) corresponds to the identity operator [1].

The formulae obtained from that of Fourier transform for the test functions in S, hold true for the fractional FT with the parameter $\alpha, \alpha$ ranging from 0 to $\pi / 2$. Moreover, since $S$ is dense in S', these formulae remain true for all functions in $\mathrm{S}$ '.

DEFINITION 2.4: Convolution: Let $\mathrm{f}, \phi \in \mathrm{G}$. The convolution of $\mathrm{f} \& \phi$ is defined as

And it is proved in [4] that ( $f * \phi)$ is in G.

DEFINITION 2.5: Fractional FT of a convolution of generalized functions : If $\mathrm{f} \in \mathrm{S}^{\prime} \& \mathrm{~g} \in \mathrm{S}$ then $R^{\alpha}(f * g)=C_{\alpha}\left(R^{\alpha} f\right)\left(R^{\alpha} h\right) \quad\left(\phi \in S \subset L^{\prime}\right)$ 


$$
(f * \phi)(z)=\frac{1}{2 \pi} \int_{\mathfrak{R}} f(z-t) \phi(t) d t
$$

i.e. $\mathrm{R}^{\alpha}(\mathrm{f} * \mathrm{~g})=\exp \left(-\mathrm{iC}_{2 \alpha} \xi^{2} \cos \alpha\right) \mathrm{R}^{\alpha}[\mathrm{f}] \mathrm{R}^{\alpha}[\mathrm{h}]$ where $\mathrm{h}=\mathrm{g} /\left(\mathrm{C}_{1 \alpha} \exp \left(\mathrm{iC}_{2 \alpha} \mathrm{x}^{2} \cos \alpha\right)\right.$.

DEFINITION 2.6: Convolution theorem for distributions: Let $\mathrm{f} \in \mathrm{S}^{\prime}\left(\mathfrak{R}^{\mathrm{n}}\right) \& \mathrm{~g} \in \mathrm{E}^{\prime}\left(\mathfrak{R}^{\mathrm{n}}\right)$.

Then $\mathrm{f} * \mathrm{~g} \in \mathrm{S}^{\prime}\left(\mathfrak{R}^{\mathrm{n}}\right) \&$ for $0<\alpha \leq \pi / 2, \quad \mathrm{R}^{\alpha}(\mathrm{f} * \mathrm{~g})=\mathrm{C}^{\prime}{ }_{\alpha}\left(\mathrm{R}^{\alpha} \mathrm{f}\right)\left(\mathrm{R}^{\alpha} \mathrm{g}\right)$

Where

$$
=C_{\alpha}^{\prime} \hat{f} \cdot \hat{g}
$$

LEMMA 2.7: For any $\mathrm{f}, \mathrm{g} \in \mathrm{G}$, for $0<\alpha \leq \pi / 2$

$$
C_{\alpha}^{\prime}=\frac{-2 C_{2 \alpha} e^{i \alpha}}{i\left(C_{1 \alpha}\right)^{2}} ; \hat{f}=\left(R^{\alpha} f\right), \hat{g}=\left(R^{\alpha} g\right)
$$

a) $\mathrm{R}^{\alpha}[\mathrm{f} * \mathrm{~g}]=\exp \left(-\mathrm{iC}_{2 \alpha} \xi^{2} \cos \alpha\right) \mathrm{R}^{\alpha}[\mathrm{f}] \mathrm{R}^{\alpha}[\mathrm{h}]$

b) $R^{\alpha}[f . g]=\exp \left(-\mathrm{iC}_{2 \alpha} \xi^{2} \cos \alpha\right)(2 \pi)^{-\mathrm{n}} \mathrm{R}^{\alpha}[\mathrm{f}] \mathrm{R}^{\alpha}[\mathrm{h}]$.

Where $\mathrm{h}=\mathrm{g} / \mathrm{C}_{1 \alpha} \exp \left(\mathrm{iC}_{2 \alpha} \mathrm{x}^{2} \cos \alpha\right)$.

DEFINITION: 2.8: Fractional FT on G: For each $\phi$ in $\mathrm{G}, 0<\alpha \leq \pi / 2$,

Where $\mathrm{K}_{\alpha}(\mathrm{t}, \mathrm{z})$ is the kernel of the transformation as given by (2). This transform is obviously will defined

$$
\hat{\phi}(z)=R^{\alpha}[\phi](z)=\frac{1}{\sqrt{2 \pi}} \int_{\Re} \phi(z) K_{\alpha}(t, z) d \Lambda---(3) .
$$

on $\mathrm{G}$.

THEOREM 2.9: For $0<\alpha \leq \frac{\pi}{2}, R^{\alpha}$ is continuous, linear, one to one mapping from $\mathrm{G}$ onto G.

LEMMA 2.10: For each $f \in G^{c}$ there are finite positive constant $\mathrm{C}$ and $\gamma$ such that for $|\langle f, \phi\rangle| \leq C\|\phi\|_{\gamma}$ for all $\phi \in \mathrm{G}$. Now, for each $f \in G^{c}, \phi \in \mathrm{G}$, we define the mapping $R^{\alpha}$ on $\Gamma$ by $\left\langle R^{\alpha}[f], \phi\right\rangle=\left\langle f, R^{\alpha}[\phi]\right\rangle, 0<\alpha \leq \frac{\pi}{2}$.

THEOREM 2.11: $R^{\alpha}, \alpha$ being a parameter; $0<\alpha \leq \frac{\pi}{2}$ is the continuous linear, one to one mapping from $G^{c}$ onto $G^{c}$.

DEFINITION 2.12: A function $\mathrm{f}$ is said to be in $\boldsymbol{G}_{\boldsymbol{n}}{ }^{\boldsymbol{c}} \quad(\mathrm{n}=0,1,2 \ldots)$ if and only if it satisfies the conditions,

(i) $\quad \mathrm{f} \in G^{c}$

(ii) $\quad$ For every $\gamma>0,\|f\|_{n, \gamma}=\sup _{z \in B_{\gamma}}\left\{e^{-n|\operatorname{Re} z|}|f(z)|\right\}$ is finite.

It is clear that $\mathrm{G}_{0}^{c} \subseteq \mathrm{G}_{1}^{c} \subseteq \ldots$ and $G^{c}=\bigcup_{0}^{\infty} \mathrm{G}_{n}^{c}$ with the family of semi norms given by

$\left\{\|\|_{n, \gamma}, \gamma>0\right\}$ Each $\mathrm{G}_{n}^{c}$ is a Frechet space and hence $G^{c}$ is a testing function space.

DEFINITION 2.13: A sequence of functions $\left\{f_{n}\right\}$ in $G^{c}$ is said to converge to a function $\mathrm{f}$ in $G^{c}$ if there exist integer $\mathrm{N}_{0}$ and $\mathrm{m}$ such that for every $\mathrm{n} \geq N_{0}, \mathrm{f}_{\mathrm{n}}, \mathrm{f}$ are in $\mathrm{G}_{m}^{c} \& \mathrm{f}_{\mathrm{n}}$ converge to $\mathrm{f}$ in $\mathrm{G}_{m}^{c}$.

DEFINITION 2.14: A sequence $\left\{f_{n}\right\}$ in $\mathrm{G}^{\prime}$ converges to $\mathrm{f}$ in $\mathrm{G}^{\prime}$ if there exists $\mathrm{C}_{\mathrm{n}}$ for $\mathrm{n}=1,2,---$ with $\mathrm{C}_{\mathrm{n}} \rightarrow 0$ as $n \rightarrow \infty$ and $\gamma>0$ such that for all $\phi \in \mathrm{G}, \quad\left|\left(f_{n}-f\right)(\phi)\right| \leq C_{n}\|\phi\|_{\gamma}$. Here after convergence in $G^{\prime}$ means the above convergence only unless stated otherwise.

DEFINITION 2.15: For $\mathrm{u} \in G^{\prime} \& \phi \in \mathrm{G}$ we define their convolution product as $(u * \phi)(z)=u\left(T_{z} \breve{\phi}\right)$ where $\breve{\phi}(t)=\phi(-t)$ and $\left(T_{z} \phi\right)(t)=\phi(t-z)$. 
THEOREM 2.16: Let $\mathrm{u} \in G^{\prime} \& \phi \in \mathrm{G}$. Then $u * \phi \in G^{c}$.

Proof: See [6] for a detailed proof.

LEMMA 2.18: Let $\mathrm{u}_{\mathrm{n}} \rightarrow 0$ as $n \rightarrow \infty$ in $G^{\prime} \& \phi \in \mathrm{G}$ be any fixed element. Then $u_{n} * \phi \rightarrow 0$ as $n \rightarrow \infty$ in $G^{c}$.

PROOF: Since $u_{n} \rightarrow 0$ as $n \rightarrow \infty$ in $G^{\prime}$ there exists $\left(c_{n}\right) \rightarrow 0$ as $n \rightarrow \infty$ and $\gamma>0$. $\left|\varphi_{n}(\phi)\right| \leq C_{n}\|\phi\|_{\gamma}, \quad(\phi \in \mathrm{G})$

Choose $\mathrm{m} \in \square$ such that $\mathrm{m}>\gamma$. We shall now prove that $\left(u_{n} * \phi\right)$ is in $\mathrm{G}_{m}^{c}$. Consider

$$
\begin{aligned}
& \left\|u_{n} * \phi\right\|_{m, \beta}=\sup _{z \in \mathbf{B}_{\beta}} e^{-m|\operatorname{Re} z|}\left|\left(u_{n} * \phi\right)(z)\right| \\
& =\sup _{z \in B_{\beta}} e^{-m|\operatorname{Re} z|}\left|u_{n}\left(T_{z} \breve{\phi}\right)\right| \\
& \leq \sup _{z \in B_{\beta}} e^{-m|\operatorname{Re} z|} C_{n}\left\|T_{z} \breve{\phi}\right\|_{\gamma} \\
& \leq \sup _{z \in B_{\beta}} C_{n} e^{(\gamma-m)|\operatorname{Re} z|}\|\phi\|_{\gamma+\beta} \\
& \leq C_{n}\|\phi\|_{\gamma+\beta}
\end{aligned}
$$

in which $\|\phi\|_{\gamma+\beta}<\infty$ and $\mathrm{C}_{n} \rightarrow 0$ as $n \rightarrow \infty$. This proves that $\left(u_{n} * \phi\right) \in \mathrm{G}_{m}^{c}$ and $\left(u_{n} * \phi\right) \rightarrow 0$ as $\mathrm{n} \rightarrow \infty$ in $\mathrm{G}_{m}^{c}$.

LEMMA 2.18: If $\mathrm{f}_{n} \rightarrow 0$ as $n \rightarrow \infty$ in $G^{c}$ then $\mathrm{f}_{n} \rightarrow 0$ as $n \rightarrow \infty G^{\prime}$.

PROOF: Since $\mathrm{f}_{\mathrm{n}} \rightarrow 0$ as $\mathrm{n} \rightarrow \infty$ in $G^{c}$ there exists $\mathrm{m}$ such that $\mathrm{f}_{\mathrm{n}} \in \mathrm{G}_{m}^{c}$ for large $\mathrm{n} \& \mathrm{f}_{\mathrm{n}} \rightarrow 0$ as $\mathrm{n} \rightarrow \infty$ in $\mathrm{G}_{m}^{c}$. For all $\gamma>0,\left\|f_{n}\right\|_{m, \gamma} \rightarrow 0$ as $n \rightarrow \infty$. Fix $\gamma>0$ and let $\mathrm{C}_{n=\left\|f_{n}\right\|_{m, \gamma}}$ so that $\mathrm{C}_{\mathrm{n}} \rightarrow 0$ as $\mathrm{n} \rightarrow \infty$.

We have $\left|f_{n}(z)\right| \leq C_{n} e^{m|\operatorname{Re} z|}$

Choose $\beta>m \& \phi \in \mathrm{G}$. Then $\left|f_{n}(\phi)\right| \leq \int_{\mathfrak{R}}\left|f_{n}(x) \phi(x)\right| d x \leq \int_{\Re} C_{n} e^{m|x|}|\phi(x)| d x$ by (5).

Since $\phi \in \mathrm{G},|\phi(x)| \leq e^{-\beta|x|}\|\phi\|_{\beta}$.

Hence $\left|f_{n}(\phi)\right| \leq \int_{\Re} C_{n} e^{(m-\beta)|x|}\|\phi\|_{\beta} d x \leq C_{n}\|\phi\|_{\beta}\left(\frac{2}{\beta-m}\right) \leq C_{n}^{\prime}\|\phi\|_{\beta}$

Where $C_{n}^{\prime}=C_{n}\left(\frac{2}{\beta-m}\right) \rightarrow 0$ as $n \rightarrow \infty$. This proves that $\mathrm{f}_{n} \rightarrow 0$ as $n \rightarrow \infty$ in $G^{\prime}$.

\section{Construction Of Boehmians \& Its Convergence Properties}

A general reference for this section can be seen in $[9,16,17]$. In this section we recall the constructions of Boehmians for two classes $B\left(G^{c}, \Delta\right)$ and $B\left(G^{\prime}, \Delta^{\prime}\right)$ from [6]. Consider the two spaces $G^{c}$ and $G$. To each pair of elements $f \in G^{c}$ and $\phi \in \mathrm{G}$, the convolution product $(f * \phi)$ defined by $(f * \phi)(z)=f\left(T_{z} \breve{\phi}\right)$ is an element of $G^{c}$ (see [6]). Thus we have a mapping * such that $*: G^{c} \times G \rightarrow G^{c}$.

It is easy to verify that this convolution product satisfies the following conditions.

(i) If $\phi, \varphi \in \mathrm{G}$ then $\phi * \varphi \in \mathrm{G}$ and $\phi * \varphi=\varphi * \phi$. 
(ii) If f $f \in G^{c} \& \phi, \varphi \in \mathrm{G}$ then $(f * \phi) * \varphi=f *(\phi * \varphi)$.

(iii) If $\mathrm{f}, \mathrm{g} \in G^{c}$ and $\phi \in \mathrm{G}$ then $(f+g) * \phi=(f * \phi)+(g * \phi)$.

Consider a class $\Delta$ of sequences $\left(\delta_{n}\right)$ in $\mathrm{G}$ satisfying the following conditions:

$$
\begin{aligned}
& \int_{\Re} \delta_{n}(x) d x=1 \\
& \int_{\mathfrak{R}}\left|\delta_{n}(x)\right| d x \leq M \\
& \lim _{n \rightarrow \infty} \int_{|x| \geq \epsilon}\left(e^{\gamma|x|}-1\right)\left|\delta_{n}(x)\right| d x=0, \forall \gamma>0 \& \in>0
\end{aligned}
$$

We shall show that the class of sequence $\Delta$ satisfies the necessary properties of "Delta sequences". Towards this, we shall prove a few preliminary results, which will help us in obtaining the required properties.

THEOREM 3.1: Let $\left(\delta_{n}\right) \in \Delta$. For each fixed $\gamma>0 \& \in>0$, the condition $\lim _{n \rightarrow \infty} \int_{|x| \geq \epsilon}\left(e^{\gamma|x|}-1\right)\left|\delta_{n}(x)\right| d x=0$ is equivalent to $\lim _{n \rightarrow \infty} \int_{\Re}\left(e^{\gamma|x|}-1\right)\left|\delta_{n}(x)\right| d x=0$.

PROOF: It suffices to prove that for each fixed $\gamma>0 \& \in>0, \lim _{n \rightarrow \infty} \int_{|x| \geq \epsilon}\left(e^{\gamma|x|}-1\right)\left|\delta_{n}(x)\right| d x=0$ tend to $\lim _{n \rightarrow \infty} \int_{\Re}\left(e^{\gamma|x|}-1\right)\left|\delta_{n}(x)\right| d x=0$. For $|x|<\eta,\left(e^{\gamma|x|}-1\right)<\left(e^{\gamma \eta}-1\right)$ and $\left(e^{\gamma \eta}-1\right) \rightarrow 0$ as $\eta \rightarrow 0$.

Hence we can choose $\eta$ such that $\left(e^{\gamma|x|}-1\right)<\frac{\epsilon}{2 M}$ with $\mathrm{M}$ as in (II). Thus

$\int_{\Re}\left(e^{\gamma|x|}-1\right)\left|\delta_{n}(x)\right| d x=\int_{|x|<\eta}\left(e^{\gamma|x|}-1\right)+\int_{|x \geq \eta|}\left(e^{\gamma|x|}-1\right)\left|\delta_{n}(x)\right| d x \quad$ in $\quad$ which $\int_{|x|<\eta}\left(e^{\gamma|x|}-1\right)\left|\delta_{n}(x)\right| d x<\frac{\epsilon}{2}$ by the choice of $\eta$ and $\int_{|x| \geq \eta}\left(e^{\gamma|x|}-1\right)\left|\delta_{n}(x)\right| d x \rightarrow 0$ as $n \rightarrow \infty$ by hypothesis. Hence $\lim _{n \rightarrow \infty} \int_{\mathfrak{R}}\left(e^{\gamma|x|}-1\right)\left|\delta_{n}(x)\right| d x=0$.

THEOREM 3.2: If $\mathrm{f} \in G^{c} \&\left(\delta_{n}\right) \in \Delta$ then $\mathrm{f} * \delta_{n} \rightarrow f$ as $n \rightarrow \infty$ in $G^{c}$.

PROOF: We have to prove that there exists m such that $\left(f * \delta_{n}-f\right) \in \mathrm{G}_{m}^{c}$ and $\left\|f * \delta_{n}-f\right\|_{m, \gamma} \rightarrow 0$ as $n \rightarrow \infty$ for each $\gamma>0$. Since $\mathrm{f} \in G^{c}, \mathrm{f} \in \mathrm{G}_{k}^{c}$ for some $\mathrm{k}$. With $\mathrm{M}$ as in (II) choose $\mathrm{m}, \mathrm{k}>k$ and $\Re$ such that $e^{(k-m) \Re}<\frac{\epsilon}{3 M}$

Given $\gamma>0$ let $\begin{aligned} A & =\left\{z \in B_{\gamma}:|\operatorname{Re} z| \leq \mathfrak{R}\right\}, \\ B & =\left\{z \in B_{\gamma}:|\operatorname{Re} z|>\mathfrak{R}\right\} .\end{aligned}$

As $\mathrm{f}$ is uniformly continuous on every compact subsets of $B_{\gamma}$, given $\frac{\epsilon}{2 M}$ there exists $\eta>0$ such that whenever $|t|<\eta$, treal and $\mathrm{z} \in A . \quad|f(z-t)-f(z)|<\frac{\epsilon}{2 M}$

Fix this $\eta$. For $|t| \geq \eta$, we have $e^{k|t|}+1 \leq K\left(e^{k^{\prime}|t|}-1\right)$ 
Where $\quad \mathrm{K}=\frac{2}{1-e^{k^{\prime} \eta}} \quad$ as $\quad$ can be easily checked. Consider

$\left\|f * \delta_{n}-f\right\|_{m, \gamma}=\sup _{z \in B_{\gamma}} e^{-m|\operatorname{Re} z|}\left|\left(f * \delta_{n}-f\right)(z)\right| \leq \sup _{z \in B_{\gamma}} e^{-m|\operatorname{Re} z|} \int_{\Re}|f(z-t)-f(z)|\left|\delta_{n}(t)\right| d t$

$\leq \sup _{z \in A} e^{-m|\operatorname{Re} z|} \int_{\Re}|f(z-t)-f(z)|\left|\delta_{n}(t)\right| d t+\sup _{z \in B} e^{-m|\operatorname{Re} z|} \int_{\Re}|f(z-t)-f(z)|\left|\delta_{n}(t)\right| d t$

$=\mathrm{J}_{1}+\mathrm{J}_{2}$ (say)

Hence $\left\|f * \delta_{n}-f\right\|_{m, \gamma} \leq J_{1}+J_{2}$

Now the integral in $J_{1}$ can be split as a sum of two integrals say $I_{1}+I_{2}$ where $I_{1}$ denotes the integration over $|t|<\eta$ and $\mathrm{I}_{2}$ denotes the integral over $|t| \geq \eta$. Using (II) and (7), it can be easily shown that $\left|I_{1}\right| \leq \frac{\epsilon}{2}$. We shall now consider $\mathrm{I}_{2}$. As $\mathrm{f} \in \Gamma_{k}^{c}$, for each $\mathrm{z} \in A$ we see that $\left|I_{2}\right|$ is dominated by $e^{-m|\operatorname{Re} z|} \int_{|t| \geq \eta}\left(e^{k|\operatorname{Re} z-t|}+e^{k|\operatorname{Re} z|}\right)\|f\|_{k, \gamma}\left|\delta_{n}(t)\right| d t$.

Hence

$$
\begin{aligned}
& \left|I_{2}\right| \leq e^{(k-m)|\operatorname{Re} z|}\|f\|_{k, \gamma} \int_{|t| \geq \eta}\left(e^{k|t|}+1\right)\left|\delta_{n}(t)\right| d t \leq e^{(k-m)|\operatorname{Re} z|}\|f\|_{k, \gamma} \int_{|t| \geq \eta} K\left(e^{k^{\prime}|t|}-1\right)\left|\delta_{n}\right| d t \text { by (8) } \\
& \leq K e^{(k-m)|\operatorname{Re} z|}\|f\|_{k, \gamma} \int_{|t| \geq \eta}\left(e^{k^{\prime}|t|}-1\right)\left|\delta_{n}(t)\right| d t \leq K\|f\|_{k, \gamma} \int_{|t| \geq \eta}\left(e^{k^{\prime}|t|}-1\right)\left|\delta_{n}(t)\right| d t
\end{aligned}
$$

Which can be made less than $\frac{\epsilon}{2}$ for sufficiently large $\mathrm{n}$ by property (III) of the family $\Delta$. Thus $\mathrm{J}_{1} \rightarrow 0$ as $n \rightarrow \infty$.

Also it is easy to see that $\mathrm{J}_{2}$ is dominated by $\sup _{z \in B} e^{-m|\operatorname{Re} z|} \int_{\Re}\left(e^{k|\operatorname{Re}(z-t)|}+e^{k|\operatorname{Re} z|}\right)\|f\|_{k, \gamma}\left|\delta_{n}(t)\right| d t$

Hence for $\mathrm{n}$ large

$$
\begin{aligned}
& J_{2} \leq \sup _{z \in B} e^{(k-m)|\operatorname{Re} z|} \int_{\Re}\left(e^{k|t|}+1\right)\left|\delta_{n}(t)\right| d t \leq \sup _{z \in B} e^{(k-m)|\operatorname{Re} z|} \int_{\Re}\left(e^{k|t|}-1+2\right)\left|\delta_{n}(t)\right| d t \\
& \leq\left(\frac{\epsilon}{3 M}(M+2 M)\right)=\in \text { by (6), (III) lemma 3.1 Thus } \mathrm{J}_{2} \rightarrow 0 \text { as } n \rightarrow \infty
\end{aligned}
$$

The theorem now follows from (9), (10) \& (11).

THEOREM 3.3: Let $\mathrm{f}, \mathrm{g} \in \Gamma^{c} \&\left(\delta_{n}\right) \in \Delta$. If $f * \delta_{n}=g * \delta_{n}, \forall n$ then fag in $\mathrm{G}^{c}$.

PROOF: Allowing $\mathrm{n}$ to tend to $\infty$ on both sides $\&$ using theorem above 3.2, we have the result.

THEOREM 3.4: Let $\left(\phi_{n}\right),\left(\varphi_{n}\right)$ be any two sequences in $\Delta$. then $\left(\phi_{n} * \varphi_{n}\right)$ is also a sequence in $\Delta$. PROOF: We shall proving (I)

$\int_{\Re}\left(\phi_{n} * \varphi_{n}\right)(x) d x=\iint_{\Re} \phi_{n}(t) \varphi_{n}(x-t) d t d x$.

The iterated integral $\iint_{\Re}\left|\phi_{n}(t)\right|\left|\varphi_{n}(x-t)\right| d x d t$ is finite as $\phi_{n}, \varphi_{n}$ are in G. Hence we can use Fubini's theorem and get $\int_{\Re}\left(\phi_{n} * \varphi_{n}\right)(x) d x=\iint_{\Re} \phi_{n}(t) \varphi_{n}(x-t) d x d t=\iint_{\Re} \phi_{n}(t) \varphi_{n}(y) d y d t$ where $\mathrm{y}=\mathrm{x}-\mathrm{t}$ $=1$ as $\left(\phi_{n}\right),\left(\varphi_{n}\right) \in \Delta$.

For proving property (II) let $\int_{\Re}\left|\phi_{n}(x)\right| d x \leq M_{1} \& \int_{\Re}\left|\varphi_{n}(x)\right| d x \leq M_{2}$. 
As before we have $\int_{\Re}\left|\left(\phi_{n} * \varphi_{n}\right)(x)\right| d x \leq M_{1} M_{2}=M$.

To prove property (III) we shall use lemma 3.1. Let $\int_{\Re}\left(e^{\gamma|x|}-1\right)\left|\phi_{n}(x)\right| d x=C_{n} \quad$ and $\int_{\Re}\left(e^{\gamma|x|}-1\right)\left|\varphi_{n}(x)\right| d x=d_{n}$. Yet another application of Fubini's theorem gives

$\int_{\Re}\left(e^{\gamma|x|}-1\right)\left(\phi_{n} * \varphi_{n}\right)(x) d x \leq \int_{\Re}\left(e^{\gamma|x|}-1\right) d x \int_{\Re}\left|\phi_{n}(x-t) \varphi_{n}(t)\right| d t$

$\leq \int_{\mathfrak{R}}\left|\varphi_{n}(t) d t\right| \int_{\mathfrak{R}}\left(e^{\gamma|x|}-1\right)\left|\phi_{n}(x-t)\right| d x \leq \int_{\mathfrak{R}}\left|\varphi_{n}(t)\right| d t \int_{\mathfrak{R}}\left(e^{\gamma|y+t|}-1\right)\left|\phi_{n}(y)\right| d y$

$\leq \int_{\mathfrak{R}}\left|\varphi_{n}(t)\right| d t \int_{\mathfrak{R}}\left(e^{\gamma|t|}\left(e^{\gamma|t|}-1\right)+\left(e^{\gamma|t|}-1\right)\right)\left|\phi_{n}(y)\right| d y$, where $\mathrm{y}=\mathrm{x}$-t. The above integral is easily seen to

be dominated by $\left(d_{n}+M_{2}\right) C_{n}+d_{n} M_{1}$, where $\mathrm{M}_{1} \& M_{2}$ are as before. This completes the proof of theorem 3.4 .

Theorem $3.3 \& 3.4$ prove that the elements of $\Delta$ satisfy the required conditions for "Delta sequences". Hence the elements of $\Delta$ can be called Delta sequences in the sense of [16]. Now in a canonical way can use $\mathrm{G}^{c}$ and $\Delta$ to constant a Boehmians space which we denote by $B\left(G^{c}, \Delta\right)$.

We now consider yet another product between elements of $G^{\prime}$ and $\mathrm{G}$ as follows. Let $\mathrm{u} \in G^{\prime}$. The product between $\mathrm{u} \& \mathrm{f}$ is an element of $G^{\prime}$ defined as (uf) $\phi=u(f \phi), \forall \phi \in \mathrm{G}$.

Let $\Delta^{\prime}$ be the class of sequences $\left(\sigma_{n}\right)$ from G such that $\Delta^{\prime}=\left\{\left(\sigma_{n}\right): \sigma_{n}=\hat{\delta}_{n}, \forall n\right.$, where, $\left.\left(\delta_{n}\right) \in \Delta\right\}$.

Here $\hat{\delta}_{n}(z)=R^{\alpha}[x](z)=\frac{1}{\sqrt{2 \pi}} \int_{\Re} \delta_{n}(t) K_{\alpha}(t, z) d \Lambda$ where $0<\alpha \leq \frac{\pi}{2}, K_{\alpha}(t, z)$ is the kernel of the transformation as given by (1).

THEOREM 3.5: For $\mathrm{u} \in G^{\prime}$ and $\left(\sigma_{n}\right) \in \Delta^{\prime}, u \sigma_{n} \rightarrow u$ as $\mathrm{n} \rightarrow \infty$ in $G^{\prime}$.

PROOF: As u $\in G^{\prime}$ by lemma 3.1 of [5] there are positive constants $\mathrm{C}$ and $\gamma>0$ such that for all $\phi \in \Gamma$,

$|u(\phi)| \leq C\|\phi\|_{\gamma}$

Choose $\beta>\gamma$. hence by (12) we can write

$\left|\left(u \sigma_{n}-u\right)(\phi)\right|=\left|u\left(\sigma_{n} \phi-\phi\right)\right| \leq C\left\|\sigma_{n} \phi-\phi\right\|_{\gamma}$

Denoting the constant function $1(\mathrm{z})=1$ by (4) we have

$\left\|\sigma_{n} \phi-\phi\right\|_{\gamma}=\sup _{z \in B_{\gamma}} e^{\gamma|\operatorname{Re} z|}\left|\left(\sigma_{n} \phi-\phi\right)(z)\right|=\sup _{z \in B_{\gamma}} e^{\gamma|\operatorname{Re} z|}|\phi(z)|\left|\left(\sigma_{n}-1\right)(z)\right| \leq \sup _{z \in B_{\gamma}} e^{(\gamma-\beta)|\operatorname{Re} z|}\|\phi(z)\|_{\beta}\left|\left(\sigma_{n}-1\right)(z)\right|$.

Hence from (13) we have $\left|\left(u \sigma_{n}-u\right)(\phi)\right| \leq C\|\phi\|_{\beta} \sup _{z \in B_{\gamma}} e^{(\gamma-\beta)|\operatorname{Re} z|}\left|\left(\sigma_{n}-1\right)(z)\right|$.

If we take $\mathrm{C}_{n}=\sup _{z \in B_{\gamma}} e^{(\gamma-\beta) \mid \operatorname{Re} z}\left|\left(\sigma_{n}-1\right)(z)\right|$ then $\left|\left(u \sigma_{n}-u\right)(\phi)\right| \leq c C_{n}\|\phi\|_{\beta}$

We shall now prove that $C_{n} \rightarrow 0$ as $n \rightarrow \infty$. as $\left(\sigma_{n}\right) \in \Delta^{\prime}, \sigma_{n}=\hat{\delta}_{n}$ for some $\left(\delta_{n}\right) \in \Delta$. hence using (I) at the appropriate place,

We have $C_{n}=\sup _{z \in B_{\gamma}} e^{(\gamma-\beta)|\operatorname{Re} z|}\left|\left(\hat{\delta}_{n}-1\right)(z)\right| \leq \sup _{z \in B_{\gamma}} e^{(\gamma-\beta)|\operatorname{Re} z|} \frac{1}{\sqrt{2 \pi}} \int_{\Re}\left|\delta_{n}(t)\right|\left|K_{\alpha}(t, z)-1\right| d \Lambda$

Let $\mathrm{R}$ be such that for a given $\in>0$ ( with $\mathrm{M}$ as in (II) )

$e^{(\gamma-\beta) R}<\frac{\epsilon}{3 M}$ 
Let $\mathrm{A}$ and $\mathrm{B}$ is defined as in Theorem 3.2. In A ( a compact set ) $\mathrm{e}^{z}$ is uniformly continuous. Hence given

$\frac{\epsilon}{2 M}$, there exists $\eta>0$ such that whenever $|t|<\eta,\left|K_{\alpha}(t, z)-1\right|<\frac{\epsilon}{2 M} \quad(\mathrm{z} \in A)$

Since $\mathrm{B}_{\gamma}$ is the disjoint union of A \& B for our purposes it is enough to show that the supremum over both A \& B tends to 0 as $\mathrm{n} \rightarrow \infty$.

Put $\mathrm{I}_{1}=\frac{1}{\sqrt{2 \pi}} \int_{|t|<\eta}\left|\sigma_{n}(t)\right|\left|K_{\alpha}(t, z)-1\right| d \Lambda$ and $I_{2}=\frac{1}{\sqrt{2 \pi}} \int_{|t| \geq \eta}\left|\delta_{n}(t)\right|\left|K_{\alpha}(t, z)-1\right| d \Lambda$.

In view of (17) and property (II) $I_{1}<\frac{\epsilon}{2}$

For $z \in A \subset B_{\gamma},\left|K_{\alpha}(t, z)-1\right| \leq\left(e^{\delta|t|}+1\right) \leq K\left(e^{\beta^{\prime}|t|}-1\right)$ for a suitable constant $\mathrm{K} \& \beta^{\prime}>\gamma$ as before. Then by property (III), $I_{2}<\frac{\epsilon}{2}$ for large n.

By (18), (19) and the fact that $\gamma<\beta$ we have

$$
\begin{aligned}
& \sup _{z \in A} e^{(\gamma-\beta)|\operatorname{Re} z|} \frac{1}{\sqrt{2 \pi}} \int_{\mathfrak{R}}\left|\delta_{n}(t)\right|\left|K_{\alpha}(t, z)-1\right| d \Lambda<\in \\
& \qquad z \in B, e^{(\gamma-\beta)|\operatorname{Re} z|} \frac{1}{\sqrt{2 \pi}} \int_{\Re}\left|\delta_{n}(t)\right|\left|K_{\alpha}(t, z)-1\right| d \Lambda \leq e^{(\gamma-\beta) R} \frac{1}{\sqrt{2 \pi}} \int_{\Re}\left|\delta_{n}(t)\right|\left|K_{\alpha}(t, z)-1\right| d \Lambda
\end{aligned}
$$

Now for

$$
\leq e^{(\gamma-\beta) R} \frac{1}{\sqrt{2 \pi}} \int_{\Re}\left|\delta_{n}(t)\right|\left(e^{\gamma|t|}+1\right) d \Lambda .
$$

As before we write $e^{\gamma|t|}+1=e^{\gamma|t|}-1+2$ and in view of (20), Property (II) of the class $\Delta$ we see that the above expression in less than or equal to $\frac{\epsilon}{3 M}(M+2 M)=\epsilon$

Hence for large $\mathrm{n}, \sup _{z \in B} e^{(\gamma-\beta)|\operatorname{Re} z|} \frac{1}{\sqrt{2 \pi}} \int_{\Re}\left|\delta_{n}(t)\right|\left|K_{\alpha}(t, z)-1\right| d \Lambda<\epsilon$

Form (15), (20) \& (21) we have $C_{n} \rightarrow 0$ as n $\rightarrow \infty$

Combining (14) \& (22) we have $u \sigma_{n} \rightarrow u$ as $n \rightarrow \infty$ in $G^{\prime}$.

THEOREM 3.6: Let $\mathrm{u}, \mathrm{v} \in \Gamma^{\prime} \&\left(\sigma_{n}\right) \in \Delta^{\prime}$. If $\mathrm{u} \sigma_{n}=v \sigma_{n}, \forall n$ then $\mathrm{u}=\mathrm{v}$ in $G^{\prime}$.

PROOF: Allowing $\mathrm{n}$ to tend to $\infty$ and using theorem 3.5 we get result.

THEOREM 3.7: If $\left(\alpha_{n}\right),\left(\beta_{n}\right) \in \Delta^{\prime}$ then $\left(\alpha_{n} \beta_{n}\right) \in \Delta^{\prime}$.

PROOF: Since $\left(\alpha_{n}\right),\left(\beta_{n}\right) \in \Delta^{\prime}$ there exists $\left(\phi_{n}\right),\left(\varphi_{n}\right) \in \Delta$ such that $\alpha_{n}=\hat{\phi}_{n} \& \beta_{n}=\hat{\varphi}_{n}, \forall n$. Hence by lemma 5.2 in [6] we get

$\alpha_{n} \beta_{n}=\hat{\phi}_{n} \hat{\varphi}_{n}=R^{\alpha}\left[\phi_{n}\right] R^{\alpha}\left[\varphi_{n}\right]=R^{\alpha}\left[\phi_{n} * \varphi_{n}\right]=\left[\phi_{n} * \varphi_{n}\right]^{\wedge}$. As $\left(\phi_{n}\right),\left(\varphi_{n}\right) \in \Delta,\left(\phi_{n} * \varphi_{n}\right) \in \Delta$ by theorem 3.4 this proves that $\left(\alpha_{n} \beta_{n}\right) \in \Delta^{\prime}$.

Theorem $3.6 \& 3.7$ prove that the elements of $\Delta^{\prime}$ satisfy the required conditions for delta sequences. Hence the sequences of $\Delta^{\prime}$ can be called "Delta sequences" in the sense of [16]. Using $G^{\prime} \& \Delta^{\prime}$, we can construct in a canonical way a Boehmians which we denote by $B\left(G^{\prime}, \Delta^{\prime}\right)$. We shall now obtain some of the convergence properties of sequences in

G\& $G^{\prime}$.

THEOREM 3.8: Let $f_{n} \rightarrow f$ as $n \rightarrow \infty$ in $\mathrm{G}^{c} \& \phi \in \mathrm{G}$ is any fixed element. Then $f_{n} * \phi \rightarrow f * \phi$ as $n \rightarrow \infty$ in $\mathrm{G}^{c}$. 
PROOF: Since $f_{n} \rightarrow f$ as $n \rightarrow \infty$ in $\mathrm{G}^{c}$, there is an $\mathrm{m} \in \mathrm{N}$ such that $\mathrm{f} \in \mathrm{G}_{m}^{c}$ and $\mathrm{f}_{\mathrm{n}} \in \mathrm{G}_{m}^{c}$ for large $\mathrm{n}$ and $\left\|f_{n}-f\right\|_{m, \gamma} \rightarrow 0$ as $n \rightarrow \infty$ in $\mathrm{G}_{m}^{c}$, for every $\gamma>0$

As $\phi \in \Gamma$, we can choose $\beta<m$ such that $|\phi(t)| \leq e^{-\beta|t|}\|\phi\|_{\beta}$.

Consider $\left\|\left(f_{n}-f\right) * \phi\right\|_{m, \gamma}=\sup _{z \in B_{\gamma}} e^{-m|\operatorname{Re} z|}\left|\left(\left(f_{n}-f\right) * \phi\right)(z)\right|$

$\left|\left(\left(f_{n}-f\right) * \phi\right)(z)\right| \leq \int_{\mathfrak{R}}\left|\left(f_{n}-f\right)(z-t)\right||\phi(t)| d t \leq \int_{\mathfrak{R}} e^{m|\operatorname{Re}(z-t)|}\left\|f_{n}-f\right\|_{m, \gamma}|\phi(t)| d t$

$\leq e^{m|\operatorname{Re} z|}\left\|f_{n}-f\right\|_{m, \gamma} \int_{\Re} e^{m|t|}|\phi(t)| d t \leq e^{m|\operatorname{Re} z|}\left\|f_{n}-f\right\|_{m, \gamma}\|\phi\|_{\beta} \int_{\mathfrak{R}} e^{(m-\beta) t \mid} d t$.

Let $\mathrm{K}=\int_{\Re} e^{(m-\beta) t \mid} d t$ then $\left|\left(\left(f_{n}-f\right) * \phi\right)(z)\right| \leq K e^{m|\operatorname{Re} z|}\left\|f_{n}-f\right\|_{m, \gamma}\|\phi\|_{\beta}$

Combining (23),(24) \& (25) We have $\left\|\left(\left(f_{n}-f\right) * \phi\right)(z)\right\|_{m, \gamma} \leq K\left\|f_{n}-f\right\|_{m, \gamma}\|\phi\|_{\beta} \rightarrow 0$ as $\mathrm{n} \rightarrow \infty$. This proves the theorem.

THEOREM 3.9: Let $f_{n} \rightarrow f$ as $\mathrm{n} \rightarrow \infty$ in $\mathrm{G}^{c}$ AND $\left(\delta_{n}\right) \in \Delta$ then $f_{n} * \delta_{n} \rightarrow f$ as $\mathrm{n} \rightarrow \infty$ in $\mathrm{G}^{c}$.

PROOF: Since $f_{n} \rightarrow f \quad$ as $n \rightarrow \infty \quad$ in $\quad \mathrm{G}^{c} \quad$ (23) hold. Also $\left(f_{n} * \delta_{n}\right)(z)-f(z)=\left(\left(f_{n}-f\right) * \delta_{n}\right)(z)+\left(f * \delta_{n}-f\right)(z)$. By theorem 3.2, $\mathrm{f} * f_{n} \rightarrow f$ as $n \rightarrow \infty$. Hence to prove the required result it is enough to prove that $\left(f_{n}-f\right) * \delta_{n} \rightarrow 0$ as $n \rightarrow \infty$ in $\mathrm{G}^{c} \cdot\left|\left(\left(f_{n}-f\right) * \delta_{n}\right)(z)\right| \leq \int_{\Re}\left|\left(f_{n}-f\right)(z-t)\right|\left|\delta_{n}(t)\right| d t$ $\leq \int_{\Re} e^{m|\operatorname{Re}(z-t)|}\left\|f_{n}-f\right\|_{m, \gamma}\left|\delta_{n}(t)\right| d t \leq e^{m|\operatorname{Re} z|}\left\|f_{n}-f\right\|_{m, \gamma}\left\{\int_{\Re}\left(e^{m|t|}-1\right)\left|\delta_{n}(t)\right| d t+\int_{\Re}\left|\delta_{n}(t)\right| d t\right\}$ $\leq e^{m|\operatorname{Re} z|}\left\|f_{n}-f\right\|_{m, \gamma}\left\{\int_{\mathfrak{R}}\left(e^{m|t|}-1\right)\left|\delta_{n}(t)\right| d t+M\right\}$ by property (II). Hence $e^{m|\operatorname{Re} z|}\left(\left(f_{n}-f\right) * \delta_{n}\right)(z) \leq M^{\prime}\left\|f_{n}-f\right\|_{m, \gamma}$ Where $M^{\prime}=M+\int_{\mathfrak{R}}\left(e^{m|t|}-1\right)\left|\delta_{n}(t)\right| d t<\infty$.

Thus $\left\|\left(f_{n}-f\right) * \delta_{n}\right\|_{m, \gamma}=\sup _{z \in B_{\gamma}} e^{-m|\operatorname{Rez}|}\left|\left(\left(f_{n}-f\right) * \delta_{n}\right)(z)\right| \leq M^{\prime}\left\|f_{n}-f\right\|_{m, \gamma} \rightarrow 0$ as $n \rightarrow \infty$ by (23) hence the theorem.

THEOREM 3.10: Let $u_{n} \rightarrow \gamma$ as $n \rightarrow \infty$ in $\mathrm{G}^{\prime} \& \varphi \in \mathrm{G}$ is any fixed element. Then $u_{n} \varphi \rightarrow u \varphi$ as $n \rightarrow \infty$ in $\mathrm{G}^{\prime}$.

PROOF: Since $u_{n} \rightarrow u$ as $n \rightarrow \infty$ in $\mathrm{G}^{\prime}$, there exists $\gamma>0 \& C_{n} \rightarrow 0$ as $n \rightarrow \infty$ such that for every $\phi \in \mathrm{G},\left|\left(u_{n} \varphi-u \varphi\right)(\phi)\right| \leq C_{n}\|\phi\|_{\gamma}$

Hence $\left|\left(u_{n} \varphi-u \varphi\right)(\phi)\right|=\left|\left(u_{n}-u\right)(\varphi \phi)\right| \leq C_{n}\|\varphi \phi\|_{\gamma}$ by (23) $\leq C_{n}\|\varphi\|_{\gamma}\|\phi\|_{\gamma} \leq C_{n}^{\prime}\|\phi\|_{\gamma}$, Where $C_{n}^{\prime}=C_{n}\|\varphi\|_{\gamma} \rightarrow 0$ as $n \rightarrow \infty$. This proves that $u_{n} \varphi=u \varphi$ as $n \rightarrow \infty$ in $\mathrm{G}^{\prime}$.

THEOREM 3.11: Let $u_{n} \rightarrow u$ as $n \rightarrow \infty$ in $\mathrm{G}^{\prime}, \&\left(\sigma_{n}\right) \in \Delta^{\prime}$. Then $u_{n} \sigma_{n} \rightarrow u$ as $n \rightarrow \infty$ in $\mathrm{G}^{\prime}$. PROOF: Since $u_{n} \rightarrow u$ as $n \rightarrow \infty$ in $\mathrm{G}^{\prime}$, (26) holds. As $\mathrm{u} \in \mathrm{G}$, there exists $\mathrm{C}$ such that for every $\phi \in \mathrm{G}$, $|u(\phi)| \leq C\|\phi\|_{\gamma}$. 
Now $\left|\left(u_{n} \sigma_{n}-u\right)(\phi)\right| \leq\left|\left(u_{n}-u\right)\left(\sigma_{n} \phi\right)\right|+\left|u\left(\sigma_{n} \phi-\phi\right)\right|$.

Also in the proof of Theorem 3.5, we have $\left\|\sigma_{n} \phi-\phi\right\|_{\gamma} \leq C_{n}\|\phi\|_{\gamma}$ for $\beta>\gamma>0 \&$

Hence $\left\|\sigma_{n} \phi\right\|_{\gamma} \leq\left(C_{n}+1\right)\|\phi\|_{\beta}$. Thus from (26),(27) \& (28) we have

$\left|\left(u_{n} \sigma_{n}-u\right)(\phi)\right| \leq C_{n}\left\|\sigma_{n} \phi\right\|_{\gamma}+C\left\|\sigma_{n} \phi-\phi\right\|_{\gamma} \leq d_{n}\|\phi\|_{\gamma}$, where $d_{n}=\left(C_{n}\left(C_{n}+C+1\right)\right)\|\phi\|_{\gamma} \rightarrow 0$

as $n \rightarrow \infty$. This proves the theorem

\section{Fractional Fourier Transform:}

Let $\left[\frac{f_{n}}{\phi_{n}}\right] \in B\left(\mathrm{G}^{c}, \Delta\right)$. Since $\mathrm{f}_{n} \in \mathrm{G}^{c}$ and $\phi \in \mathrm{G}$, form [4,7] we know that $\hat{f}_{n}=R^{\alpha}\left[f_{n}\right] \in$ $\mathrm{G}^{\prime}$ and $\hat{\phi}_{n}=R^{\alpha}\left[\phi_{n}\right] \in \mathrm{G}$. As $\left(\phi_{n}\right) \in \Delta,\left(\hat{\phi}_{n}\right) \in \Delta^{\prime}$. Also $\frac{f_{n}}{\phi_{n}}$ is a quotient in $\quad B\left(\mathrm{G}^{c}, \Delta\right)$ implies that $f_{m} * \phi_{n}=f_{n} * \phi_{m}, \forall m, n \in \square . \mathrm{N}$ Taking the fractional $\mathrm{FT}$ on both sides we have $R^{\alpha}\left(f_{m} * \phi_{n}\right)=R^{\alpha}\left(f_{n} * \phi_{m}\right), \forall m, n \in \square \mathrm{N}$ in G' $($ see $[2,3])$

I.e. $C_{\alpha}^{\prime} R^{\alpha}\left(f_{m}\right) R^{\alpha}\left(\phi_{n}\right)=C_{\alpha}^{\prime} R^{\alpha}\left(f_{n}\right) R^{\alpha}\left(\phi_{m}\right), \forall m, n \in \square \mathrm{N}$ where $C_{\alpha}^{\prime}=\frac{2 C_{2 \alpha} e^{i \alpha}}{i\left(C_{1 \alpha}\right)^{2}} \quad$ (see [7])

This proves that $\frac{R^{\alpha}\left(f_{n}\right)}{R^{\alpha}\left(\phi_{n}\right)}=\frac{\hat{f}_{n}}{\hat{\phi}_{n}}$ is a quotient in $B\left(\mathrm{G}^{\prime}, \Delta^{\prime}\right)$.

Hence whenever $\left[\frac{f_{n}}{\phi_{n}}\right] \in B\left(\mathrm{G}^{c}, \Delta\right),\left[\frac{\hat{f}_{n}}{\hat{\phi}_{n}}\right] \in B\left(\mathrm{G}^{c}, \Delta\right)$.

Now we are ready to define the fractional Fourier transform on $B\left(\mathrm{G}^{c}, \Delta\right)$.

DEFINITION 4.1: Let $\mathrm{x}=\left[\frac{f_{n}}{\phi_{n}}\right] \in B\left(\mathrm{G}^{c}, \Delta\right)$. The fractional Fourier transform $\hat{x}=R^{\alpha}[x]$ of $\mathrm{x}$ is defined, as Boehmians in $B\left(\mathrm{G}^{\prime}, \Delta^{\prime}\right)$ by $\hat{x}=\left[\frac{\hat{f}_{n}}{\hat{\phi}_{n}}\right]$ where $\hat{f}_{n}, \hat{\phi}_{n}$ are the classical fractional FT of $\mathrm{f}_{n}, \phi_{n}$ in $\mathrm{G}^{\prime}, \mathrm{G}$ respectively.

THEOREM 4.2: The fractional Fourier transform on $B\left(\mathrm{G}^{c}, \Delta\right)$ is well defined.

PROOF: Let $\left[\frac{f_{n}}{\phi_{n}}\right]=\left[\frac{g_{n}}{\varphi_{n}}\right]$ in $B\left(\mathrm{G}^{c}, \Delta\right)$. Then $f_{m} * \varphi_{n}=g_{n} * \phi_{m}, \forall m, n \in \square . \mathrm{N}$ and taking

Fractional Fourier transform we have $R^{\alpha}\left[f_{m} * \varphi_{n}\right]=R^{\alpha}\left[g_{n} * \phi_{m}\right]$

$C_{\alpha}^{\prime} R^{\alpha}\left[f_{m}\right] R^{\alpha}\left[\varphi_{n}\right]=C_{\alpha}^{\prime} R^{\alpha}\left[g_{n}\right] R^{\alpha}\left[\phi_{m}\right]$

$C_{\alpha}^{\prime} \hat{f}_{m} \hat{\varphi}_{n}=C_{\alpha}^{\prime} \hat{g}_{n} \hat{\phi}_{m}$ in G', $\forall m, n \in \square$ N i.e. $\hat{f}_{m} \hat{\varphi}_{n}=\hat{g}_{n} \hat{\phi}_{m}$.

This proves that $\left[\frac{\hat{f}_{n}}{\hat{\phi}_{n}}\right]=\left[\frac{\hat{g}_{n}}{\hat{\varphi}_{n}}\right]$ in $B\left(\mathrm{G}^{\prime}, \Delta^{\prime}\right)$.

THEOREM 4.3: The Fractional Fourier transform from $B\left(\mathrm{G}^{c}, \Delta\right)$ to $B\left(\mathrm{G}^{\prime}, \Delta^{\prime}\right)$ is one-one and onto. 
PROOF: If Fractional FT $\hat{x}=\left[\frac{\hat{f}_{n}}{\hat{\phi}_{n}}\right]=0$ in $B\left(\mathrm{G}^{\prime}, \Delta^{\prime}\right)$ then $\hat{f}_{n}=0, \forall n$ in $\mathrm{G}^{\prime}$, which implies that $f_{n}=0, \forall \mathrm{n}$ in $\mathrm{G}^{\prime}$. Thus $f_{n} * \phi_{n}=0$ in $\mathrm{G}^{c}$, for every $\mathrm{n}$ in $B\left(\mathrm{G}^{c}, \Delta\right)$, $\left[\frac{f_{n}}{\phi_{n}}\right]=\left[\frac{f_{n} * \phi_{n}}{\phi_{n} * \phi_{n}}\right]$. Hence $\mathrm{x}=\left[\frac{f_{n}}{\phi_{n}}\right]=0$ in $B\left(\mathrm{G}^{c}, \Delta\right)$. This proves that the transform is one-to-one. Let $y=\left[\frac{g_{n}}{\hat{\varphi}_{n}}\right] \in B\left(\mathrm{G}^{\prime}, \Delta^{\prime}\right)$. Since $\mathrm{g}_{n} \in \mathrm{G}^{\prime}$, there exists $\mathrm{f}_{n} \in \mathrm{G}^{\prime}$ such that $g_{n}=\hat{f}_{n}, \forall$ n. Hence $y=\left[\frac{\hat{f}_{n}}{\hat{\varphi}_{n}}\right]$. Since $\mathrm{f}_{n} \in \mathrm{G}^{\prime}$ and $\varphi_{n} \in \mathrm{G}, \quad\left(f_{n} * \varphi_{n}\right)$ is in $\mathrm{G}^{c}$ and hence $x=\left[\frac{f_{n} * \varphi_{n}}{\varphi_{n} * \varphi_{n}}\right] \in B\left(\mathrm{G}^{c}, \Delta\right)$. Now $\hat{x}=\left[\frac{\hat{f}_{n} \hat{\varphi}_{n}}{\hat{\varphi}_{n} \hat{\varphi}_{n}}\right]=\left[\frac{\hat{f}_{n}}{\hat{\phi}_{n}}\right]=y$ in $B\left(\mathrm{G}^{\prime}, \Delta^{\prime}\right)$.

Hence for a given $y=\left[\frac{g_{n}}{\hat{\phi}_{n}}\right] \in B\left(\mathrm{G}^{\prime}, \Delta^{\prime}\right)$, there exists $x=\left[\frac{f_{n} * \varphi_{n}}{\varphi_{n} * \varphi_{n}}\right] \in B\left(\mathrm{G}^{c}, \Delta\right)$

(With $\hat{f}_{n}=g_{n}$ ) such that $\hat{x}=y$. This proves that fractional FT from $B\left(\mathrm{G}^{c}, \Delta\right)$ to $B\left(\mathrm{G}^{\prime}, \Delta^{\prime}\right)$ is onto.

THEOREM 4.4: The fractional FT from $B\left(\mathrm{G}^{c}, \Delta\right)$ to $B\left(\mathrm{G}^{\prime}, \Delta^{\prime}\right)$ is an extension of the classical fractional FT on $\mathrm{G}^{\prime}$.

PROOF: $u \in \mathrm{G}^{\prime} \Rightarrow \hat{u}=R^{\alpha}[u] \in \mathrm{G}^{\prime}$ (see[7]). By theorem $\mathrm{u} \in \mathrm{G}^{\prime}$ can be identified with the Boehmians $\left[\frac{u * \delta_{n}}{\delta_{n}}\right]$ in $B\left(\mathrm{G}^{c}, \Delta\right)$ where $\left(\delta_{n}\right)$ is any delta sequence in $\Delta$.

Let $x=\left[\frac{u * \delta_{n}}{\delta_{n}}\right]$. Then $\hat{x}=R^{\alpha}[x]=\left[\frac{\hat{u} \hat{\delta}_{n}}{\hat{\delta}_{n}}\right]$ which is identified with $\hat{u}$ in $B\left(\mathrm{G}^{\prime}, \Delta^{\prime}\right)$ by theorem 4.1 this proves the theorem.

Note: $\mathrm{G}$ ' can be considered as a subspace of both these spaces.

THEOREM 4.5: The fractional FT is linear transform from $B\left(\mathrm{G}^{c}, \Delta\right)$ to $B\left(\mathrm{G}^{\prime}, \Delta^{\prime}\right)$.

PROOF: Take $\mathrm{x}=\left[\frac{f_{n}}{\phi_{n}}\right]$ and $y=\left[\frac{g_{n}}{\varphi_{n}}\right]$ in $B\left(\mathrm{G}^{c}, \Delta\right)$.

Then $x+y=\left[\frac{\left(f_{n} * \varphi_{n}\right)+\left(g_{n} * \phi_{n}\right)}{\phi_{n} * \varphi_{n}}\right] \in B\left(\mathrm{G}^{c}, \Delta\right)$.

Now $[x+y]^{\wedge}=R^{\alpha}(x+y)=R^{\alpha}\left[\frac{\left(f_{n} * \varphi_{n}\right)+\left(g_{n} * \phi_{n}\right)}{\phi_{n} * \varphi_{n}}\right]=\frac{R^{\alpha}\left(f_{n} * \varphi_{n}\right)+R^{\alpha}\left(g_{n} * \phi_{n}\right)}{R^{\alpha}\left(\phi_{n} * \varphi_{n}\right)}$

$=\frac{R^{\alpha}\left(f_{n}\right) R^{\alpha}\left(\varphi_{n}\right)+R^{\alpha}\left(g_{n}\right) R^{\alpha}\left(\phi_{n}\right)}{R^{\alpha}\left(\phi_{n}\right) R^{\alpha}\left(\varphi_{n}\right)}=\left[\frac{\hat{f}_{n} \hat{\varphi}_{n}+\hat{g}_{n} \hat{\phi}_{n}}{\hat{\phi}_{n} \hat{\varphi}_{n}}\right]=\left[\frac{\hat{f}_{n}}{\hat{\phi}_{n}}\right]+\left[\frac{\hat{g}_{n}}{\hat{\varphi}_{n}}\right]=\hat{x}+\hat{y}$ in $B\left(\mathrm{G}^{\prime}, \Delta^{\prime}\right)$.

Let $\gamma \in \square$ and consider $\gamma x=\left[\frac{\gamma f_{n}}{\phi_{n}}\right]$ in $B\left(\mathrm{G}^{c}, \Delta\right)$ 
$(\gamma x)^{\wedge}=R^{\alpha}(\gamma x)=R^{\alpha}\left[\frac{\gamma f_{n}}{\phi_{n}}\right]=\left[\frac{\gamma \hat{f}_{n}}{\hat{\phi}_{n}}\right]=\gamma \hat{x}$ in $B\left(\mathrm{G}^{\prime}, \Delta^{\prime}\right)$. This shows that Fourier transform is linear transform from $B\left(\mathrm{G}^{c}, \Delta\right)$ to $B\left(\mathrm{G}^{\prime}, \Delta^{\prime}\right)$.

DEFINITION 4.6: Let $\mathrm{x}=\left[\frac{f_{n}}{\phi_{n}}\right]$ in $B\left(\mathrm{G}^{c}, \Delta\right)$ and $\varphi \in \mathrm{G}$.

The convolution of $\mathrm{x}$ and $\varphi$ is defined in $B\left(\mathrm{G}^{c}, \Delta\right)$ as $x * \varphi=\left[\frac{f_{n} * \varphi}{\phi_{n}}\right]$.

THEOREM 4.7: Let $x \in B\left(\mathrm{G}^{c}, \Delta\right)$ and $\varphi \in \mathrm{G}$ be any fixed element. Then $(x * \varphi)^{\wedge}=\hat{x} \hat{\varphi}$ in $B($ $\mathrm{G}^{\prime}, \Delta^{\prime}$ ) where $\hat{\varphi}$ is the classical fractional FT of $\varphi$ in $\mathrm{G}$.

PROOF: If $\mathrm{x}=\left[\frac{f_{n}}{\phi_{n}}\right]$ in $B\left(\mathrm{G}^{c}, \Delta\right)$ then $x * \varphi=\left[\frac{f_{n} * \varphi}{\phi_{n}}\right]$.

Hence $(x * \varphi)^{\wedge}=R^{\alpha}(x * \varphi)=R^{\alpha}(x) R^{\alpha}(\varphi)=\left[\frac{\hat{f}_{n} \hat{\varphi}}{\hat{\phi}_{n}}\right]=\left[\frac{\hat{f}_{n}}{\hat{\phi}_{n}}\right] \hat{\varphi}=\hat{x} \hat{\varphi}$ in $B\left(\mathrm{G}^{\prime}, \Delta^{\prime}\right)$.

THEOREM 4.8: Let $\left(x_{n}\right)$ be a sequence of Boehmians $\delta$ - converging to $\mathrm{x}$ in

$B\left(\mathrm{G}^{c}, \Delta\right)$. Then $\left(\hat{x}_{n}\right)$ is a sequence of Boehmians $\delta$-converging to $\hat{x}$ in $B\left(\mathrm{G}^{\prime}, \Delta^{\prime}\right)$ i.e. Fractional FT is a continuous map form $B\left(\mathrm{G}^{c}, \Delta\right)$ to $B\left(\mathrm{G}^{\prime}, \Delta^{\prime}\right)$.

PROOF: Since $x_{n} \rightarrow x$ as $n \rightarrow \infty$ i.e. $B\left(\mathrm{G}^{c}, \Delta\right)$, there exists a delta sequence $\left(\delta_{k}\right) \in \Delta$ such that $\left(x_{n} * \delta_{k}\right),\left(x * \delta_{k}\right) \in \mathrm{G}^{c}$ and $x_{n} * \delta_{k} \rightarrow x * \delta_{k}$ as $n \rightarrow \infty$ in $\mathrm{G}^{c} \forall k \in \square . \mathrm{N}$

By the definition $x_{n} * \delta_{k} \rightarrow x * \delta_{k}$ as $n \rightarrow \infty$ in $\mathrm{G}^{\prime} \forall k \in \square . \mathrm{N}$

Since $\left(x_{n} * \delta_{k}\right),\left(x * \delta_{k}\right) \in \mathrm{G}^{c}$

$$
\left(x_{n} * \delta_{k}\right),\left(x * \delta_{k}\right) \in \mathrm{G}^{\prime}
$$

As fractional FT is continuous in $\mathrm{G}^{\prime}$, (see [7]). We have $\left(x_{n} * \delta_{k}\right)^{\wedge}=R^{\alpha}\left(x_{n} * \delta_{k}\right) \rightarrow R^{\alpha}\left(x * \delta_{k}\right)=\left(x_{n} * \delta_{k}\right)^{\wedge}$ as $n \rightarrow \infty$ in $\mathrm{G}^{\prime} \forall k \in \square . \mathrm{N}$

By theorem 4.5 we have $\hat{x}_{n} \hat{\delta}_{k} \rightarrow \hat{x} \hat{\delta}_{k}$ as $n \rightarrow \infty$ in $\mathrm{G}^{\prime} \forall k \in \square . \mathrm{N}$

Which proves that $\hat{x}_{n} \stackrel{\delta}{\longrightarrow} \hat{x}$ as $n \rightarrow \infty B\left(\mathrm{G}^{\prime}, \Delta^{\prime}\right)$.

\section{Conclusion:}

In this paper we introduced two spaces of Boehmians each of which contains the dual of a certain space of entire functions. Both these spaces of Boehmians are shown to be isomorphic to each other under the Fractional Fourier transform. We extended the theory of the Fractional Fourier transform on this new Boehmians space.

\section{References}

[1] Alieva, T. and Barbe, A.M, Fractional Fourier analysis of objects with scaling symmetry in the book: Fractals in engineering, ed.J. Lavyvehel, E.luton and C. Tricot, Springer Verlag, (1997), 252-265. Allain, C. and Cloitre, M.: Physics Rew E 33, (1986), 3566-3569.

[2] Bhosale, B.N., Topics in the Theory of integral Transformations of generalized Functions, Doctoral Thesis, University of Kolhapur, India (2001).

[3] Bhosale, B.N.and Chaudhary, M.S., Fractional Fourier Transform of Distributions of Compact support, Bull. Cul. Math. Soc. 94, No. 5, (2002), 349-358.

[4] Howell, K.B., A new theory of Fourier analysis, part I, The space of test functions, J. Math. Anal. Appl. 168, (1992), 342-350.

[5] Howell, K.B., A new theory of Fourier analysis, part II, Further analysis on space of test functions, J. Math. Anal. Appl. 173, (1993), 419-429. 
[6] Howell, K.B., A new theory of Fourier analysis, part III, Basic analysis on the dual space, J. Math. Anal. Appl. 175, (1993), 257-267.

[7] Howell, K.B., A new theory of Fourier analysis, part IV, Basic multiplication and Convolution on dual spaces J. Math. Anal. Appl. 168, (1998), 342-350.

[8] V. Karunakaran, Generalized Integral Transforms, International workshop \& seminar on Trans. Techniques \& their Appl., Kerala, (2001), 13-16.

[9] V. Karunakaran \& R. Roopkumar, Ultra Boehmians \& their Fourier Transforms, Fractional Calculus \& Applied Ana., An International J. for theory \& Appl., Vol. 5, No. 2, (2002), 181-194.

[10] V. Karunakaran \& N.V. Kalpakam, Boehmians \& Fourier Transform, Integral Transforms \& Special Functions, Vol. 9, No.3, (2000), 197-216.

[11] P. Mikusinski, Fourier Transform for Integrable Boehmians, Rocky Mountain J.Math., 17, (1987), 577-582.

[12] P. Mikusinski, Tempered Boehmians and Ultra distribution, Proc. of Amer.Math. Soc., 123, No. 3, (1995), 813-817.

[13] P. Mikusinski \& Ahmed Zayed, On the extension of Zak transform, The J. of methods of applications of Analysis, Vol.2, (1995), 160-172

[14] P. Mikusinski \& Ahmed Zayed, The Radon Transform on Boehmians, Proc. of Amer. Math. Soc. 118, No.2, (1993), 561 570 .

[15] P. Mikusinski, A. Morse \& D. Nemzer, The two sided Laplace Transform for Boehmians, Integral Transforms \& Special functions, Vol. 2, No.3, (1994), 219-230.

[16] P. Mikusinski \& J. Mikusinski, Quotients de suites et leurs applications dans l'analysis fonctionnelle, C.R. Acad. Sc. Paris, 293, series I, (1981) 463-464.

[17] P. Mikusinski, Convergence of Boehmians, Japan J. Math. 9, (1983), 159-179

[18] P. Mikusinski, Boehmians \& Generalized functions, Acta. Math. Hung., 51, (1998), 197-216

[19] P. Mikusinski \& D. Nemzer, The Laplace transforms on a class of Boehmians, Bull. Austral. Math. Soc., 46, (1992), 347-352.

[20] P. Mikusinski \& E.R Dill, Strong Boehmians, Proc. of Amer. Math. Soc., 119, No.3, (1998), 885-888

[21] P. Mikusinski, The Fourier transform of tempered Boehmians, Fourier Analysis, Lecture notes in Pure \& Appl. Math. Marcel Dekker, New York, (1994), 303-309.

[22] R.S. Pathak, Integral Transformations of generalized functions \& their applications, Gordon \& Breach Sc. Publ., Netherland.

[23] R.S. Pathak, Generalized functions \& their appl. Plenum press, New York (1993), 141- 147.

[24] G.E. Shilov \& I.M. Gelfand, Generalized function, 1-3, Acad. Press, New York (1964). 